\title{
METÁSTASIS ESPLÉNICA SOLITARIA EN UNA PACIENTE CON ADENOCARCINOMA COLORRECTAL*
}

\author{
Drs. Marta de la Fuente-Bartolomé ${ }^{1}$, Carlos Piedracoba-Cadahia ${ }^{2}$, \\ Alejando Moreno-Bargueiras ${ }^{1}$, Mercedes Pérez-Carreras ${ }^{2}$, \\ Alfredo Ibarra-Peláez ${ }^{3}$, Carlos Morales-Gutiérrez ${ }^{3}$ \\ ${ }^{1}$ Cirugía General y del Aparato Digestivo A. Hospital Universitario 12 de Octubre. \\ 2 Medicina del Aparato Digestivo. Hospital Universitario 12 de Octubre. \\ 3 Cirugía General y Digestivo. Clínica Ruber. \\ Madrid, España.
}

\begin{abstract}
\section{Splenic metastases secondary to colorrectal carcinoma}

Introduction: Splenic metastases are unusual, arising in less than $1 \%$ of all metastases. Splenic metastases from colorrectal carcinoma is considered excepcional. If present, they generally occur in concert with disseminated disease. Case report: We present a case of 78 year old man operated of colon tumor by right hemicolectomy. Nine months after first surgical, CT scan showed metastases in spleen, splenectomy was performed.
\end{abstract}

Key words: Splenic metastases, colorrectal carcinoma.

\section{Resumen}

Introducción: Las metástasis esplénicas son inusuales, representando menos del 1\% de todas las metástasis. Que este tipo de localización secundaria sea ocasionado por carcinomas colorrectales puede considerarse como algo excepcional. Cuando se presentan generalmente lo hacen en el contexto de una enfermedad diseminada. Caso clínico: Presentamos el caso de un varón de 78 años de edad que fue intervenido de un carcinoma colorrectal mediante hemicolectomía derecha. Nueve meses después de la primera cirugía el escáner muestra metástasis en el bazo, por lo que se realizó una esplenectomía.

Palabras clave: Metástasis esplénica, carcinoma colorrectal.

*Recibido el 11 de septiembre de 2014 y aceptado para publicación el 11 de diciembre de 2014.

Los autores no refieren conflictos de interés.

Aceptado y presentado en el XIV Congreso de la Sociedad Canaria de Cirugía, que tendrá lugar en Tenerife los días 14 a 16 de noviembre de 2014, con la colaboración de la sección española del Colegio Internacional de Cirujanos.

Correspondencia: Dra. Marta de la Fuente Bartolomé

martilladelafuente@gmail.com 


\section{Introducción}

Las metástasis esplénicas (ME) constituyen el 1\% de los tumores malignos del bazo ${ }^{1}$. Habitualmente suceden en el contexto de una neoplasia con afectación multivisceral y/o carcinomatosis peritoneal siendo excepcional su aparición como metástasis única.

De acuerdo con diferentes series de autopsias, las ME aparecen en el $2-7 \%$ de sujetos fallecidos por tumores malignos, generalmente con origen en la mama, el pulmón, el colon, el recto o el ovario ${ }^{2-4}$.

Se han descrito ME en el 4,5\% de pacientes con carcinoma colorrectal (CCR), la mayoría múltiples y metacrónicas. La aparición de una ME única con origen en un CCR es excepcional, sólo existen 26 casos descritos en la literatura científica anglosajona ${ }^{1}$.

\section{Caso clínico}

Presentamos el caso de una paciente con un CCR y una ME aislada y metacrónica: Mujer de 78 años, sin antecedentes de interés, con síndrome constitucional y anemia microcítica, a la que se realiza colonoscopia visualizando una lesión en colon derecho a la que se toma muestras, con diagnóstico anatomopatológico de adenocarcinoma colorrectal. En el estudio de extensión no hay enfermedad diseminada. Se realiza hemicolectomía derecha oncológica con resultado anatomopatológico de la pieza de adenocarcinoma (pT3N1Mx), con quimioterapia adyuvante. En la revisión de los 12 meses se detecta una elevación del marcador tumoral Antígeno Carcinoembrionario (CEA) (37 ng/mL), junto con la aparición en el TC de una lesión sólida en el bazo de $23 \mathrm{~mm}$ (Figura 1) que se confirma mediante RMN (Figura 2), siendo no patológica según el FDG-PET. Tres meses después el CEA es de $98 \mathrm{ng} / \mathrm{ml}$, no hay cambios en la lesión esplénica en el TC, pero presenta ya captación sospechosa en el FDG-PET. Se realiza una segunda laparotomía con esplenectomía, confirmándose el diagnóstico de $\mathrm{ME}$. La paciente ha permanecido asintomática y sin datos de recidiva hasta la actualidad.

\section{Discusión}

El CCR se asocia a metástasis en el $20 \%$ de los casos, que suelen localizarse en el hígado, ganglios regionales, pulmones y peritoneo ${ }^{5,6}$. Sin embargo, la aparición de metástasis esplénicas es excepcional. Se desconocen los mecanismos por los que puede producirse la diseminación tumoral desde el colon hasta el bazo y los motivos por los que este hecho es

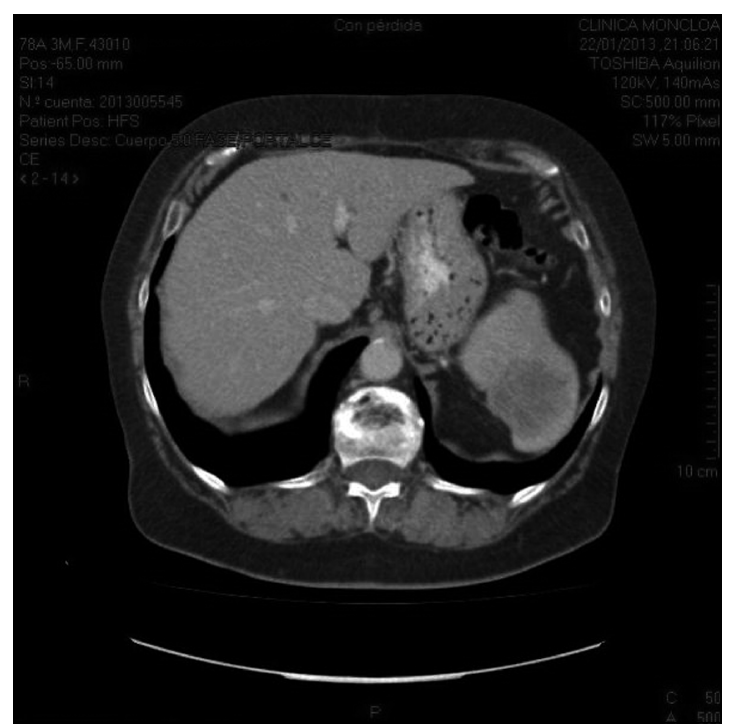

Figura 1. Corte transversal de TC donde se aprecia lesión sólida esplénica.

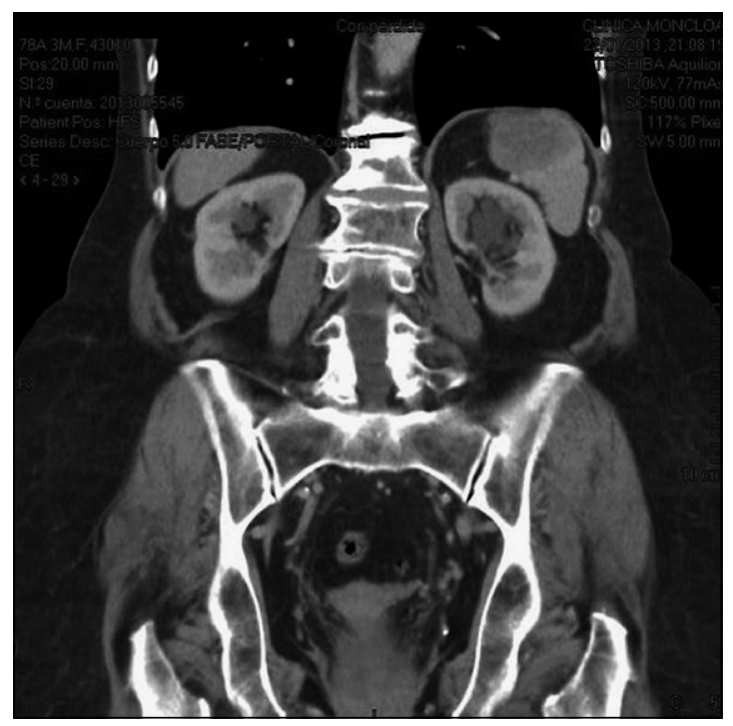

Figura 2. Corte coronal de TC donde se aprecia lesión sólida esplénica.

$\tan$ infrecuente. Puesto que el bazo carece de ganglios linfáticos aferentes, la hipótesis más aceptada es la que propone 2 posibles vías de diseminación: la diseminación por contigüidad, en el caso de la carcinomatosis peritoneal, y la hematógena, especialmente en el caso de las metástasis únicas (vía vasos esplénicos y vena mesentérica superior e inferior ${ }^{1,7}$.

Abi Saad et al., han resumido en una publicación reciente los 26 casos de pacientes con CCR y 
metástasis esplénicas comunicados en la literatura anglosajona durante las últimas cinco décadas ${ }^{1}$. De acuerdo con esta serie, en la mayoría de los casos la ME es asintomática, múltiple, metacrónica (tras un intervalo entre 3 y 44 meses) y secundaria a un tumor primario localizado en el colon descendente. Generalmente, las ME no producen síntomas, aunque en ocasiones producen una esplenomegalia dolorosa y, en casos graves, rotura esplénica.

El diagnóstico de ME puede sospecharse cuando se observa un aumento de los valores plasmáticos del CEA y/o aparecen lesiones esplénicas en las pruebas de imagen durante el seguimiento oncológico. Aunque el TC y la resonancia magnética son las más utilizadas, el FDG-PET-TC ha demostrado mayor sensibilidad, siendo de gran valor en aquellos casos que sean dudosos, antes de tomar una decisión terapéutica ${ }^{8,9}$.

Existe consenso en cuanto a que la esplenectomía es el tratamiento de primera elección, especialmente en el caso de ME única ${ }^{9}$. Aunque se prefiere la intervención por laparotomía, cada vez existe más experiencia y mejores resultados de la cirugía tumoral por laparoscopia, por lo que es posible que en algunos casos pueda accederse por esta vía ${ }^{8,10}$, aunque hasta el momento no se ha demostrado.

En el caso que comunicamos, la ME fue asintomática y apareció 12 meses después del diagnóstico del CCR, sin asociarse a enfermedad metastásica en otros órganos. La sospecha diagnóstica se estableció por el aumento del CEA y la aparición de una lesión sólida en el bazo durante el seguimiento con TC, sospechosa de maligna en el FDG-PET-TC. La esplenectomía confirmó el diagnóstico de ME única y fue curativa. En este caso la localización del tumor primario fue el colon derecho, alcanzando el bazo probablemente vía hematógena, a través de las venas mesentérica superior y esplénica.

Hemos creído interesante comunicar este nuevo caso de ME solitaria de un CCR con el fin de aportar información que permita obtener mayor evidencia científica en el manejo de estos pacientes.

\section{Referencias}

1. Abi Saad GS, Hussein M, El-Saghir NS, Termos S, Sharara AI, Shamseddine A. Isolated splenic metastasis from colorectal cancer.Int J Clin Oncol 2011;16:306-13. doi:10.1007/s10147-010-0182-2. Epub 2011 Jan 22.

2. Berge T. Splenic metastases: frequencies and patterns. Acta Pathol Micro-biol Scand.1974;82:499-506.

3. Kraus MD, Fleming MD, Vonderheide RH. The spleen as a diagnostic specimen: a review of 10 years' experience at two tertiary care institutions. Cancer 2001;91:2001-9.

4. Compérat E, Bardier-Dupas A, Camparo P, Capron F, Charlotte F. Splenic metastases. Clinicopathologic Presentation, Differential Diagnosis, and Pathogenesis. Arch Pathol Lab Med 2007;131:965-9.

5. Pedersen IK, Burcharth F, Roikjaer O, Baden H. Resection of liver metastases from colorectal cancer. Indications and results. Dis Colon Rectum 1994;37:1078-82.

6. Jemal A, Siegel R, Ward E, Hao Y, Xu J, Thun MJ. Cancer statistics, 2009. CA Cancer J Clin. 2009;59:225-49.

7. Indudhara R, Vogt D, Levin HS, Church J. Isolated splenic metastases from colon cancer. South Med J. 1997;90:633-6.

8. Cavallaro A, Modugno P, Specchia M, Pontenza AE, Loschiavo V, Colli R, et al. Isolated splenic metastasis from colon cancer. J Exp Clin Cancer Res. 2004;23:1436.

9. Montemurro S, Maselli E, Ruggieri E, Caliandro C, Rucci A, Zito AF, et al. Isolated splenic metastasis from colon cancer. Report of a case. Tumori 2008; 94:422-5.

10. Warren S, Davis AH. Studies on tumor metastasis: the metastases of carcinoma to the spleen. Am J Cancer 1934;21:517-3. 